\title{
Neutron stars within the Bogoliubov quark-meson coupling model
}

Rabhi, Aziz; Providência, Constança; Moszkowski, Steven A.; Da Providência, João; Bohr, Henrik

Published in:

Physical Review C

Link to article, DOI:

10.1103/PhysRevC.103.035811

Publication date:

2021

Document Version

Publisher's PDF, also known as Version of record

Link back to DTU Orbit

Citation (APA):

Rabhi, A., Providência, C., Moszkowski, S. A., Da Providência, J., \& Bohr, H. (2021). Neutron stars within the Bogoliubov quark-meson coupling model. Physical Review C, 103(3), [035811].

https://doi.org/10.1103/PhysRevC.103.035811

\section{General rights}

Copyright and moral rights for the publications made accessible in the public portal are retained by the authors and/or other copyright owners and it is a condition of accessing publications that users recognise and abide by the legal requirements associated with these rights.

- Users may download and print one copy of any publication from the public portal for the purpose of private study or research.

- You may not further distribute the material or use it for any profit-making activity or commercial gain

- You may freely distribute the URL identifying the publication in the public portal 


\title{
Neutron stars within the Bogoliubov quark-meson coupling model
}

\author{
Aziz Rabhi $\odot,{ }^{1,2, *}$ Constança Providência $\odot,{ }^{1, \dagger}$ Steven A. Moszkowski, ${ }^{3, \ddagger}$ João da Providência,,${ }^{1}{ }^{\S}$ and Henrik Bohr ${ }^{4, \|}$ \\ ${ }^{1}$ CFisUC, Department of Physics, University of Coimbra, 3004-516 Coimbra, Portugal \\ ${ }^{2}$ University of Carthage, Avenue de la République BP 77-1054 Amilcar, Tunisia \\ ${ }^{3}$ UCLA, Los Angeles, California 90095, USA \\ ${ }^{4}$ Department of Physics, B.307, Danish Technical University, DK-2800 Lyngby, Denmark
}

(Received 18 August 2020; revised 26 January 2021; accepted 8 March 2021; published 24 March 2021; corrected 5 April 2021)

\begin{abstract}
A quark-meson coupling model based on the quark model proposed by Bogoliubov for the description of the quark dynamics is developed and applied to the description of neutron stars. Starting from a SU(3) symmetry approach, it is shown that this symmetry has to be broken in order to satisfy the constraints set by the hypernuclei and by neutron stars. The model is able to describe observations such as two-solar-mass stars or the radius of canonical neutron stars within the uncertainties presently accepted. If the optical potentials for $\Lambda$ and $\Xi$ hyperons in symmetric nuclear matter at saturation obtained from laboratory measurements of hypernuclei properties are imposed, the model predicts no strangeness inside neutron stars.
\end{abstract}

DOI: 10.1103/PhysRevC.103.035811

\section{INTRODUCTION}

The study of nuclear matter properties has received, in the recent few decades, much attention. Such investigations are particularly important in connection with nuclearastrophysics. The recent detection of the gravitational waves GW170817 and the follow-up of the electromagnetic counterpart from a neutron-star (NS) merger [1-3], together with the simultaneous measurement of the radius and mass of the pulsar PSR J0030-0451 by NICER [4,5] are very important observations to constrain the equation of state (EoS) of dense matter. Besides, the two-solar-mass pulsars PSR J1614-2230 [6,7], PSR J0348 + 0432 [8], and MSP J0740 + 6620 [9] are also setting important constraints on the nuclear matter EoS at high densities. In particular, these large masses put some difficulties on the possible existence of non-nucleonic degrees of freedom, such as hyperons or quark matter, in the inner core of the NS. In Ref. [6], it was even suggested that PSR J1614-2230 would rule out the appearance of these degrees of freedom inside pulsars. Since then many works have shown that the present existing constraints on the high density EoS are sufficiently weak to still allow for the onset of hyperons, quarks, or other non-nucleonic degrees of freedom inside twosolar-mass neutron stars [10-16].

In relativistic mean field (RMF) models [17-20], the nucleon-nucleon interaction is described in terms of the coupling of nucleons, assumed to be point particles, with isoscalar scalar mesons, isoscalar vector mesons, and isovector vector

\footnotetext{
*rabhi@uc.pt

${ }^{\dagger}$ cp@uc.pt

†Deceased.

§providencia@uc.pt

"hbohr@dtu.dk
}

mesons. To describe adequately nuclear matter properties, RMF models include both self- and cross-interactions $[18,19]$ among these mesons or density-dependent couplings [20]. There have been attempts, based on the MIT bag model [21] and on the Nambu-Jona-Lasinio (NJL) model [22], to take into account the quark structure of the nucleon, in order to incorporate the meson couplings at a more basic level. Along these lines, the nuclear equation of state $(\mathrm{EoS})$ has been obtained and the properties of nuclear matter have been determined by Guichon, Saito, and Thomas [21,23-25] and by others [26-28] in the framework of quark-meson coupling (QMC) models. Still within the same model, in Refs. [29,30], the authors have studied the effect of strong magnetic fields on kaon condensation and hyperonic matter, respectively. An improved version of the QMC model based on the MIT bag model, which includes the polarizability of the nucleons and uses Hartree-Fock, has been proposed in Refs. [25,31,32]. More recently, nuclear matter has also been investigated in the context of a modified QMC model based on the replacement of the nucleon bag by an independent quark potential [33-36]. In this model, confinement is described by including an equally mixed scalar-vector harmonic potential and extra corrections that take into account chiral symmetry restoration and gluon exchange.

In QMC models the hyperon parameters are fixed to the hyperon masses $[24,27,28,31,32]$. The coupling of the $\sigma$ meson to hyperons is obtained self-consistently, while the $\omega$-hyperon coupling is fixed by symmetry arguments or by imposing that accepted values of the hyperon potential in symmetric nuclear matter at saturation are reproduced. This last procedure is the one that has also been adopted in Ref. [36] for the modified quark meson coupling model (MQMC) model. In this model the incompressibility depends on the quark mass and only taking a quark mass above $200 \mathrm{MeV}$, it is possible to describe neutron stars containing hyperons in their core with masses 
above $1.9 M_{\odot}$. In Refs. [31,32] the use of a Hartree-Fock approach and the explicit introduction of a polarizability term in the hyperon description pushes the onset of hyperons to densities close to $4 \rho_{0}$. Both in the above QMC and MQMC models the $\sigma, \omega$, and $\rho$ mesons couple exclusively to the $u$ and $d$ quarks, corresponding to a perfect $\omega-\phi$ mixing.

Motivated by the idea of the string tension, Bogoliubov proposed an independent quark model for the description of the quark dynamics [37]. The phenomenological description of hadronic matter in the spirit of the QMC approach, combined with Bogoliubov's interesting quark model, has been considered in Ref. [38] for nonstrange matter and in Ref. [39] for strange matter. We refer to the model considered in Refs. [38,39] as the Bogoliubov-QMC model. This model assumes that baryons are composed of quarks bound by a linearly raising potential, as suggested by gauge theories. The constituent mass of the $u, d$ quarks is generated dynamically. In the present study, we consider a generalization of the model proposed in Ref. [39], where the couplings of the quarks $s$ to vector bosons have not been explicitly considered. Instead, in Ref. [39] it is postulated that the couplings of hyperons to the vector mesons are well constrained by the phenomenological hyperon potentials in nuclear matter, a procedure similar to the one undertaken in Refs. [28,36]. In the following, the consequences of considering the coupling of the quarks $u, d, s$ to appropriate vector bosons starting from a SU(3) symmetry approach are explicitly investigated. We discuss under which conditions it is possible to describe two-solar-mass stars with a nonzero strangeness content and determine their chemical content. In particular, we conclude that this symmetry has to be broken in order to satisfy the constraints set by the hypernuclei and by neutron stars.

In Sec. II we briefly present the model, in Sec. III the description of hadronic matter with strangeness is introduced and the $\beta$-equilibrium equation of state is built. In the Sec. IV, we obtain the structure and properties of neutron stars described by the present models and discuss the results. Finally some concluding remarks are drawn in the last section.

\section{THE MODEL}

We consider the Hamiltonian

$$
h_{D}=-i \boldsymbol{\alpha} \cdot \nabla+\beta\left(\kappa|\mathbf{r}|+m-g_{\sigma}^{q} \sigma\right) \text {. }
$$

Here, $m$ is the current quark mass, $\beta$ and the components $\alpha_{x}$, $\alpha_{y}, \alpha_{z}$ of $\boldsymbol{\alpha}$ are Dirac matrices, $\sigma$ denotes the external scalar field, $g_{\sigma}^{q}$ denotes the coupling of the quark to the $\sigma$ field, and $\kappa$ denotes the string tension:

$$
\begin{array}{rlr}
\beta & =\left[\begin{array}{cc}
I & 0 \\
0 & -I
\end{array}\right], \quad \alpha_{x}=\left[\begin{array}{cc}
0 & \sigma_{x} \\
\sigma_{x} & 0
\end{array}\right], \quad \alpha_{y}=\left[\begin{array}{cc}
0 & \sigma_{y} \\
\sigma_{y} & 0
\end{array}\right], \\
\alpha_{z} & =\left[\begin{array}{cc}
0 & \sigma_{z} \\
\sigma_{z} & 0
\end{array}\right],
\end{array}
$$

where $\sigma_{x}, \sigma_{y}, \sigma_{z}$ are the Pauli matrices. The current quark mass $m$ is taken to be $m=0$ for $u, d$ quarks because their constituent mass is assumed to be determined exclusively by the value of $\kappa$. The constituent mass of the $u, d$ quarks is generated dynamically, while the constituent mass of the $s$ quark arises both dynamically and from its "current" mass. Considering an SU(3) symmetry, the coupling $g_{\sigma}^{q}$ is assumed to be the same for quarks $u, d, s$. The eigenvalues of $h_{D}$ are obtained by a scale transformation from the eigenvalues of

$$
h_{D_{0}}=-i \boldsymbol{\alpha} \cdot \nabla+\beta(|\mathbf{r}|-a) .
$$

The parameter $a$ is related to the nucleon radius. We need the lowest positive eigenvalue of $h_{D_{0}}$. We cannot apply the variational principle to $h_{D_{0}}$, because its eigenvalues are not bounded from below, but we can apply the variational principle to the square of the Hamiltonian,

$$
h_{D_{0}}^{2}=-\nabla^{2}+(|\mathbf{r}|-a)^{2}+i \beta \boldsymbol{\alpha} \cdot \frac{\mathbf{r}}{|\mathbf{r}|} .
$$

We wish to determine variationally the lowest positive eigenvalue of $h_{D_{0}}$ versus $a$. The variational ansatz should take into account the Dirac structure of the quark wave function, so that we consider the following ansatz:

$$
\Psi_{b, \lambda}=\left[\begin{array}{c}
\chi \\
i \lambda(\boldsymbol{\sigma} \cdot \mathbf{r}) \chi
\end{array}\right] e^{-(|r|-a-b)^{2} / 2},
$$

where $b, \lambda$ are variational parameters, and $\chi$ is a two-spinor. From the wave function in Eq. (3), it is clear that $a+b$ is a measure of the nucleon radius. Minimizing the expectation value of $h_{D 0}^{2}$ for $\Psi_{b, \lambda}$, the following expression for the quark mass is found:

$$
\begin{aligned}
\frac{m^{2}(\kappa, a)}{\kappa} & =\min _{\lambda, b} \frac{\left\langle\psi_{b, \lambda}\left|h_{D 0}^{2}\right| \psi_{b, \lambda}\right\rangle}{\kappa\left\langle\psi_{b, \lambda} \mid \psi_{b, \lambda}\right\rangle} \\
& =\min _{\lambda, b} \frac{\mathcal{K}_{0}+\mathcal{V}_{0}+\mathcal{V}_{01} \lambda+\left(\mathcal{K}_{1}+\mathcal{V}_{1}\right) \lambda^{2}}{\mathcal{N}_{0}+\mathcal{N}_{1} \lambda^{2}}
\end{aligned}
$$

where $\mathcal{N}_{0}, \mathcal{V}_{0}, \mathcal{K}_{0}, \mathcal{N}_{1}, \mathcal{V}_{1}$, and $\mathcal{K}_{1}$ are all given in Ref. [38].

Minimization of Eq. (4) with respect to $\lambda$ is readily performed, so that

$$
\begin{aligned}
\frac{m^{2}(\kappa, a)}{\kappa}= & \frac{1}{2} \min _{b}\left[\frac{\mathcal{K}_{0}+\mathcal{V}_{0}}{\mathcal{N}_{0}}+\frac{\mathcal{K}_{1}+\mathcal{V}_{1}}{\mathcal{N}_{1}}\right. \\
& \left.-\sqrt{\left(\frac{\mathcal{K}_{0}+\mathcal{V}_{0}}{\mathcal{N}_{0}}-\frac{\mathcal{K}_{1}+\mathcal{V}_{1}}{\mathcal{N}_{1}}\right)^{2}+\left(\frac{\mathcal{V}_{01}}{\sqrt{\mathcal{N}_{0} \mathcal{N}_{1}}}\right)^{2}}\right]
\end{aligned}
$$

Minimization of the right-hand side (r.h.s.) of Eq. (5) with respect to $b$ may be easily implemented. We have found that, in the interval $-1.25<a<2.4$, which covers the range of densities we consider, we may express the ground-state energy $m(\kappa, a)$ of $h_{D 0}$ with sufficient accuracy as

$$
\begin{aligned}
\frac{m(\kappa, a)^{2}}{\kappa}= & 2.64123-2.35426 a+0.825225 a^{2} \\
& -0.072244 a^{3}-0.0314736 a^{4}+0.00155171 a^{5} \\
& +0.00257144 a^{6}
\end{aligned}
$$

Taking $a=g_{\sigma}^{q} \sigma / \sqrt{\kappa}$ for quarks $u, d$, we get, in the vacuum, the constituent mass of these quarks equal to $313 \mathrm{MeV}$, with $a=0$ and $\kappa=37106.931784 \mathrm{MeV}^{2}$. For 
the quark $s, a=a_{s}=-1.2455+g_{\sigma}^{q} \sigma / \sqrt{\kappa}$ reproduces the vacuum constituent mass $504 \mathrm{MeV}$ of this quark. Consequently, the mass $M_{B}^{*}$ of the baryon $B$ is given as follows:

$$
\begin{aligned}
& M_{N}^{*}=M_{P}^{*}=3 m(\kappa, a), \quad M_{\Lambda}^{*}=2 m(\kappa, a)+m\left(\kappa, a_{s}\right), \\
& M_{\Xi}^{*}=m(\kappa, a)+2 m\left(\kappa, a_{s}\right) .
\end{aligned}
$$

As we discuss in the following, the $\Sigma$ hyperons will not be considered because experimental data seem to indicate that the potential of the $\Sigma$ hyperon in nuclear matter is quite repulsive [40], so that their appearance is disfavored.

\section{HADRONIC MATTER}

To describe hadronic matter, we introduce the vectorisoscalar $\omega$ meson and the vector-isovector $b_{3}$ meson and use nuclear matter properties to fix the couplings of these mesons to nucleons.

In the present model, the field $\omega$ is replaced by a vector field of the $\eta$ type, in the spirit of Ref. [41], with structure $[\bar{u} u+\bar{d} d+(1+\delta) \bar{s} s] /\left[2+(1+\delta)^{2}\right]^{1 / 2}$, where $1+\delta>0$, so that the coupling of the $\omega$ meson to the quark $s$ is equal to the coupling to the quarks $u, d$ multiplied by $1+\delta$. This ansatz breaks the SU(3) symmetry and, as shown later, stiffens the EoS. The parameter $\delta$ will be fixed by the potential $U_{\Lambda}$ of the $\Lambda$ hyperon in symmetric nuclear matter at saturation and by imposing the existence of two-solar-mass stars.

In this framework, the energy density is given by

$$
\mathcal{E}=\frac{\gamma}{(2 \pi)^{3}}\left(\sum_{B,(B \neq \Sigma)} \int^{k_{F_{B}}} d^{3} k \sqrt{k^{2}+M_{B}^{* 2}}+\sum_{l} \int^{k_{F_{l}}} d^{3} k \sqrt{k^{2}+M_{l}^{2}}\right)+\frac{1}{2} m_{\sigma}^{2} \sigma^{2}+\frac{1}{2} m_{\omega}^{2} \omega^{2}+\frac{1}{2} m_{b_{3}}^{2} b_{3}^{2},
$$

and the thermodynamical potential is given by

$$
\Phi=\frac{\gamma}{2 \pi^{2}}\left\{\sum_{B,(B \neq \Sigma)} \int^{k_{F_{B}}} k^{2} d k\left[\sqrt{k^{2}+M_{B}^{* 2}}-\left(\mu-q_{B} \lambda\right)\right]+\int^{k_{F_{l}}} k^{2} d k\left(\sqrt{k^{2}+M_{l}^{2}}-\lambda\right)\right\}+\frac{1}{2} m_{\sigma}^{2} \sigma^{2}+\frac{1}{2} m_{\omega}^{2} \omega^{2}+\frac{1}{2} m_{b_{3}}^{2} b_{3}^{2},
$$

where the Lagrange multiplier $\mu$ controls the baryon density and $\lambda$ the electrical charge. The $\sigma$ field is determined from the minimization of $\Phi$ with respect to $\sigma$,

$$
\frac{\partial \Phi}{\partial \sigma}=0
$$

The coupling of the scalar meson $\sigma$, which, in the spirit of SU(3) flavor symmetry, is the same for all quarks, is encapsulated into the definition of $M_{B}^{*}$, Eq. (7). The sources of the fields $\omega$ and $b_{3}$ (respectively, $\rho_{0}$ and $\rho_{3}$ ) are given by

$$
\rho_{0}=\frac{\gamma}{(2 \pi)^{3}} \sum_{B,(B \neq \Sigma)} \zeta_{B} \int^{k_{F B}} d^{3} k, \quad \rho_{3}=\frac{\gamma}{(2 \pi)^{3}} \sum_{B,(B \neq \Sigma)} \eta_{B} \int^{k_{F B}} d^{3} k,
$$

with

$$
\begin{aligned}
& \zeta_{P}=\zeta_{N}=1, \quad \zeta_{\Lambda}=1+\delta, \quad \zeta_{\Xi_{0}}=\zeta_{\Xi_{-}}=1+2 \delta, \\
& \eta_{P}=1, \quad \eta_{N}=-1, \quad \eta_{\Lambda}=0, \quad \eta_{\Xi_{0}}=1, \quad \eta_{\Xi_{-}}=-1 .
\end{aligned}
$$

The relation between the fields and the respective sources is given by

$$
\omega=\frac{3 g_{\omega}^{q} \rho_{0}}{m_{\omega}^{2}}, \quad b_{3}=\frac{g_{b_{3}}^{q} \rho_{3}}{m_{b_{3}}^{2}} .
$$

We start by fixing the free parameter $\kappa$ of the Bogoliubov model. This is obtained by fitting the nucleon mass $M=939 \mathrm{MeV}$. Next, the desired values of the neutron effective mass $M^{*} / M=0.773$, nuclear matter binding energy $E_{B}=\epsilon / \rho_{B}-M_{N}=-15.7 \mathrm{MeV}$, the incompressibility $K=$ $315.0 \mathrm{MeV}$, in agreement with the range of values proposed in Ref. [42], and the nucleon radius $R_{B}=0.1163 \mathrm{fm}$ at saturation density, $\rho_{B}=0.145 \mathrm{fm}^{-3}$, are obtained by setting $g_{\sigma}^{q}=$ 4.0539996 and $3 g_{\omega}^{q}=g_{\omega N}^{q}=9.2474196$. The coupling constant $g_{b_{3}}^{q}=3.9532889$ is fixed in order to have the symmetry energy coefficient $a_{4}=29 \mathrm{MeV}$ and the symmetry energy slope $L=79.45 \mathrm{MeV}$, at saturation density. The value we consider for $L$ is well inside the range of values obtained in Ref. [43] from a huge number of experimental data and astrophysical observations, $L=58.7 \pm 28.1 \mathrm{MeV}$. We have chosen a slightly low saturation density in order that the model produces reasonable values of incompressibility $K$.

There is an appreciable mass difference between the hyperons $\Lambda$ and $\Sigma$, which, according to Refs. [44-46] is due to a hyperfine splitting. Moreover, it should be kept in mind that the $\mathrm{SU}(2)$ symmetry is a very important one. The hyperon $\Lambda$ is an isosinglet; the nucleon and the $\Xi$ are isodoublets; the $\Sigma$ is 


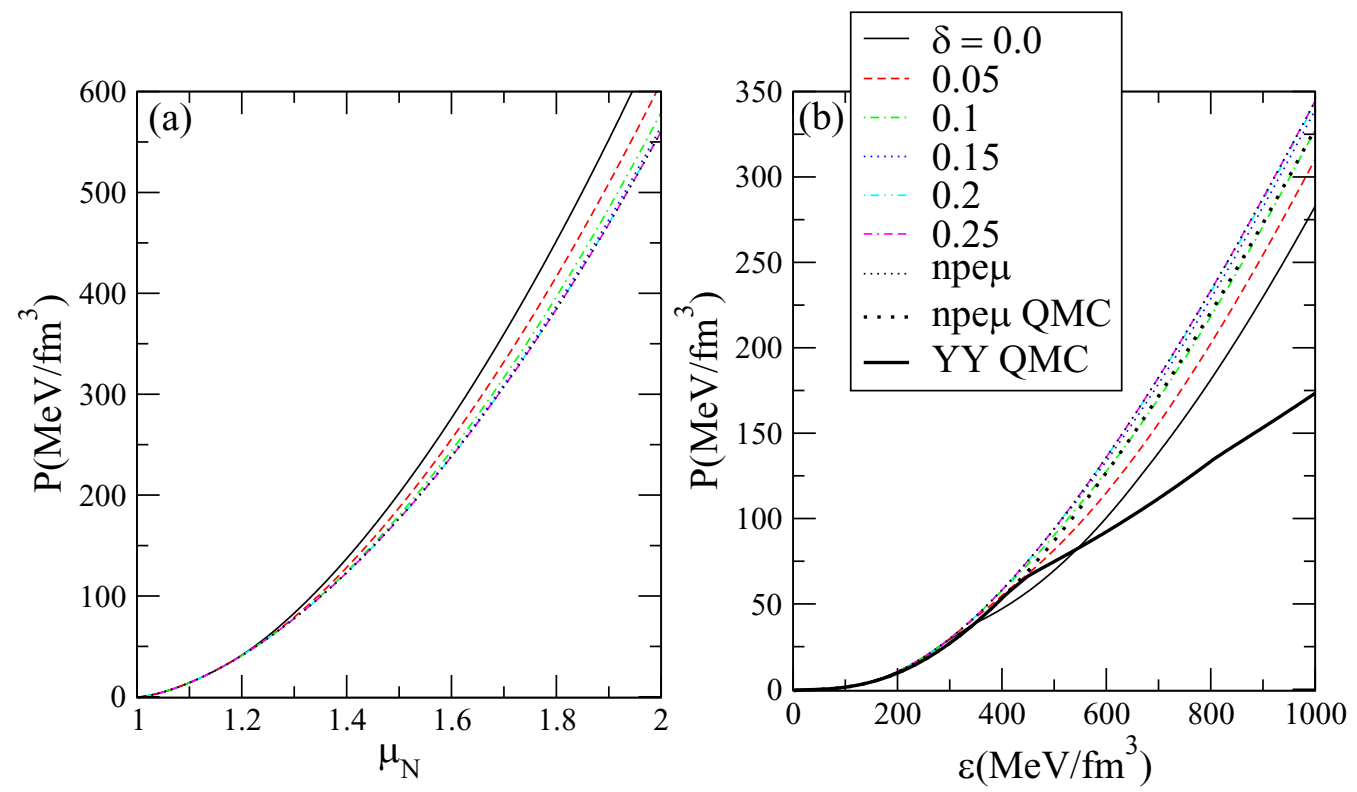

FIG. 1. Pressure versus neutron chemical potential comparing neutron, proton, and leptonic matter with hyperonic matter for $\delta=0.0,0.05$, $0.1,0.15,0.2$, and 0.25 (left panel), and the pressure versus energy density for $\beta$-equilibrium nucleonic and hyperonic matter compared with the corresponding QMC EoS obtained in Ref. [28]. The thick lines have been obtained with QMC models [28] and all the thin lines represent EoS obtained for the Bogoliubov-QMC model with and without hyperons.

an isotriplet. In addition, it is known that the $\Sigma$-nucleus potential in symmetric nuclear matter seems to be repulsive [40,47]. Our nucleon model does not take into account the mechanism responsible for the above-mentioned hyperfine splitting, the $\Sigma$ and $\Lambda$ hyperons are degenerate, and besides also leads to an attractive optical potential for the $\Sigma$. We overcome this problem by omitting the $\Sigma$ in sums over $B$, as explicitly indicated in Eq. (11), and in analogous sums in the sequel. We are only performing sums over baryons which are either isosinglets or isodoublets. The omission of the $\Sigma$ hyperon is in accordance with the general result obtained when a repulsive $\Sigma$ potential in symmetric matter at saturation density of the order of $30 \mathrm{MeV}$ is considered [11,12,16]: $\Sigma$ hyperons are not present inside neutron stars.

Minimization of $\Phi$ with respect to $k_{F_{B}}$ leads to

$$
\sqrt{k_{F B}^{2}+M_{B}^{* 2}}+3 g_{\omega}^{q} \omega \zeta_{B}+g_{b_{3}}^{q} b_{3} \eta_{B}=\mu-q_{B} \lambda .
$$

The quantity $\mu-q_{B} \lambda$ is usually referred to as the chemical potential of baryon $B$. Minimization of $\Phi$ with respect to $k_{F_{e}}$ leads to

$$
\sqrt{k_{F e}^{2}+M_{e}^{2}}=\lambda,
$$

so the Lagrange multiplier $\lambda$ is usually cal led the electron Fermi energy.

Explicitly, for $N, \Lambda, \Xi$, Eq. (14) reduces to

$$
\begin{aligned}
\sqrt{k_{F_{N}}^{2}+M_{N}^{* 2}}+3 g_{\omega}^{q} \omega+g_{b_{3}}^{q} b_{3} \eta_{N} & =\mu-q_{N} \lambda, \\
\sqrt{k_{F_{\Lambda}}^{2}+M_{\Lambda}^{* 2}}+3 g_{\omega}^{q}(1+\delta) \omega & =\mu, \\
\sqrt{k_{F_{\Xi}}^{2}+M_{\Xi}^{* 2}}+3 g_{\omega}^{q}(1+2 \delta) \omega+g_{b_{3}}^{q} b_{3} \eta_{\Xi} & =\mu-q_{\Xi} \lambda .
\end{aligned}
$$

Then, according to the prescription of Ref. [48], we have

$$
\begin{aligned}
& U_{\Lambda}:=M_{\Lambda}^{*}-M_{\Lambda}+3 g_{\omega}^{q}(1+\delta) \omega, \\
& U_{\Xi}:=M_{\Xi}^{*}-M_{\Xi}+3 g_{\omega}^{q}(1+2 \delta) \omega,
\end{aligned}
$$

and it is possible to fix the coupling to the quark $s$ in such a way that a reasonable $U_{\Lambda}$ is obtained. We find that a small change in $\delta$ leads to big changes in $U_{\Lambda}$ and $U_{\Xi}$. However, the EoS is almost insensitive to the value of $\delta$ for a wide range of values of $U_{\Lambda}$ around the proper one. This model predicts a competition between negatively charged hyperons and leptons. This is natural in view of Bodmer-Witten's conjecture [49,50], according to which the ground state of baryonic matter at high densities should involve only quarks $u, d$, s, without leptons.

The pressure $P$ obtained for different values of $\delta$ is shown in Fig. 1 as a function of the baryonic chemical potential $\mu_{N}$ (left panel) and the energy density (right panel). In the right panel we also include the EoS obtained with the QMC model [28] for comparison. The inclusion of hyperons softens the EoS as expected, corresponding to a larger pressure for a given chemical potential. When the $\delta$ parameter is turned on, the EoS gets stiffer and, for the range of the chemical potential shown, the EoS with $\delta \geqslant 0.2$ almost coincides with the nucleonic EoS. The QMC EoS softens strongly above $3 \rho_{0}$ and becomes much softer than the Bogoliubov-QMC EoS, even with $\delta=0$.

In Fig. 2, the fractions of baryons and leptons for $\beta$ equilibrium matter are represented as a function of density for different values of the parameter $\delta$. For $\delta=0$ the onset of hyperons occurs for $\rho=2 \rho_{0}$, and the first hyperon to set in is the $\Lambda$ and at a slightly larger density the $\Xi^{-}$. A finite $\delta$ pushes the onset of these particles to larger densities, and 


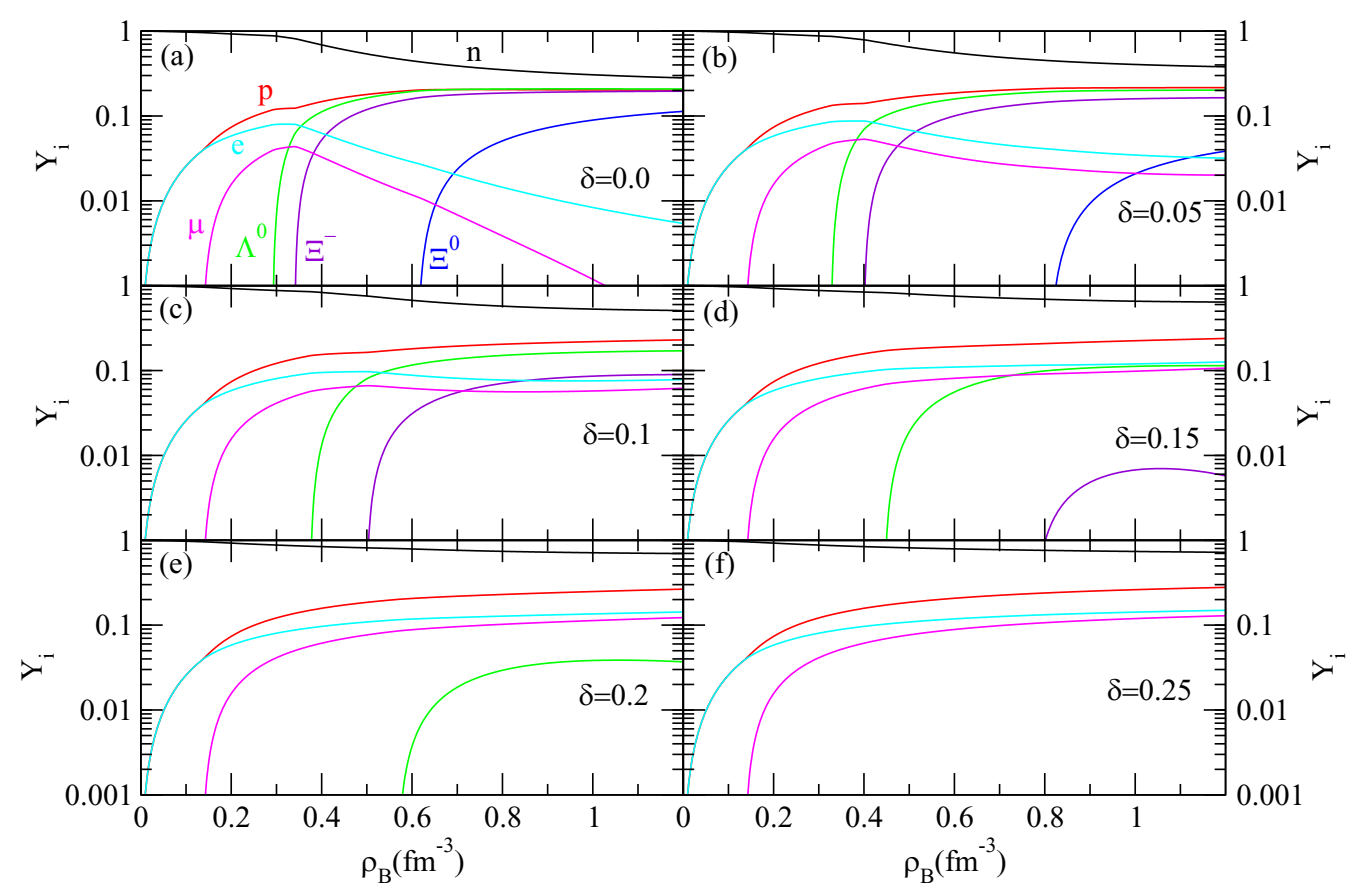

FIG. 2. Baryonic and leptonic particle fractions as a function of the baryonic density, for several values of the parameter $\delta$. For $\delta=0.25$, the onset of hyperons is shifted to densities above $1.2 \mathrm{fm}^{-3}$. The central baryonic density lies between 0.9 and $1.1 \mathrm{fm}^{-3} \mathrm{depending}$ on the hyperonic content.

for $\delta=0.2$ the onset of $\Lambda$ occurs at $\approx 4 \rho_{0}$ and the $\Xi^{-}$above $8 \rho_{0}$. Besides the fraction of $\Lambda \mathrm{s}$ in this last scenario never goes above $4 \%$ while for $\delta=0$ it reaches $20 \%$.

To study the structure of neutron stars described by the present model we have integrated the Tolman-OppenheimerVolkoff equations for spherical stars in equilibrium [51,52]. The complete EoS was obtained by matching the BaymPethcik-Sutherland EoS for the outer crust [53], and the inner crust was obtained within a Thomas-Fermi description of the nonhomogeneous matter for the NL $3 \omega \rho$ model with the symmetry energy slope at saturation equal to $77 \mathrm{MeV}$ [54], to the core EoS. It has been discussed in Ref. [16] that a nonunified EoS, i.e., a neutron-star EoS constituted by a crust and core EoS obtained from different models, gives rise to an uncertainty in the radius of low-mass stars. For the crust, two distinct contributions are included: the outer crust and the inner crust. While the first is relatively well constrained, the second is clearly dependent on the underlying model used to describe the star. In the present work we consider the conclusion drawn in Ref. [54] where it was shown that taking an inner crust EoS from a model with a similar dependence of the symmetry energy on the density would predict realistic radii. The complete EoS that has been used to integrate the TOV equations is plotted in the left panel of Fig. 3 on a $\log -\log$ scale so that it is clearly seen that the crust-core transition is smooth. In Table I, several star properties are given, including the maximum gravitational mass $M_{\max }$, and the corresponding baryonic mass $M_{\max }^{b}$, radius $R$, energy density and baryonic density at the center $E_{0}$, and $\rho^{c}$, the radius of $1.4 M_{\odot}$ and $1.6 M_{\odot}$ stars, $R_{1.4}$ and $R_{1.6}$, and the hyperon potentials in symmetric nuclear matter at saturation $U_{\Lambda}\left(\rho_{0}\right)$ and $U_{\Xi}\left(\rho_{0}\right)$.
For $\delta=0$, we find that the EoS does not describe stars with masses above 1.92 solar masses. For $\delta \geqslant 0.2$, the EoS and the curve mass vs radius are almost insensitive to the value of $\delta$. The onset of hyperons occurs at a density above $\approx 0.6 \mathrm{fm}^{-3}$ and the hyperon fraction is too small. Let us point out that we obtain reasonable values for the hyperon potentials in symmetric nuclear matter for $\delta \approx 0.25$, see Table I. With this value of $\delta$ no hyperons will appear inside neutron stars. A similar conclusion was obtained by Ref. [55] within a microscopic approach that includes three-body contributions of the form $N N Y$. Under these results, two-solar-mass stars will not contain hyperons because they will set in at densities of the order of the neutron star central density or above. At this point let us add a comment on the hyperon content within other QMC models. In Refs. [27,28] hyperons have been included in the QMC model [23]. Taking different choices for the meson-hyperon couplings, the onset of hyperons occurs below $3 \rho_{0}$. Due to the softening the EoS with the hyperon onset the maximum star mass decreases from $\approx 2.2 M_{\odot}$ to $\approx 1.8 M_{\odot}$, see Fig. 3(b) where the nucleonic $M / R$ curve is plotted for the nucleonic EoS (thick dotted line) and the hyperonic EoS (thick full line). Including a nonlinear $\omega \rho$ term that allows us to soften the density dependence of the symmetry energy pushes the hyperon onset to densities above $3 \rho_{0}$ and increases the maximum star mass to $\approx 1.9 M_{\odot}$. Considering the improved QMC model [25], Stone et al. have also included hyperons [31,32]. This version of the model, which takes into account the color hyperfine interaction and the scalar polarizability of the baryons, and besides considers the Hartree-Fock approximation, predicts that hyperons set in close to $4 \rho_{0}$ and maximum star masses lie in the interval $\approx 1.9 M_{\odot}-2 M_{\odot}$. Even though, in smaller amount, hyperons do set in inside neutron 

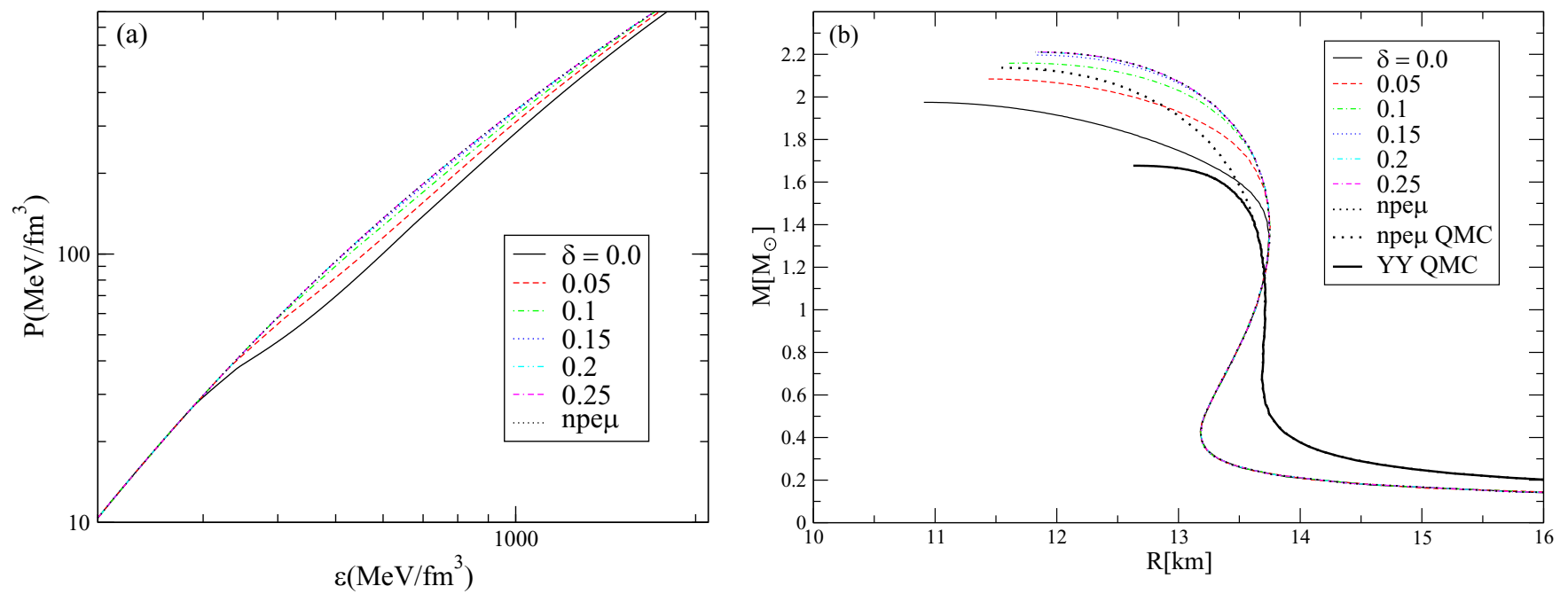

FIG. 3. EoS (left) and mass-radius curves obtained from the integration of the TOV equations (right), for different values of the $\delta$ parameter. The curves stop at the maximum-mass configuration. The family of stars for nucleonic stars constituted by npe $\mu$ matter is also represented. Also shown are the mass-radius curves obtained with the QMC model in Ref. [28] for comparison (thick full and dotted lines).

stars. Within the MQMC model [36] the EoS is quite sensitive to the quark mass and only by taking a quark mass above 200 $\mathrm{MeV}$ is it possible to describe neutron stars with masses above $1.9 M_{\odot}$ containing small amounts of hyperons in their core.

The canonical star with a mass $1.4 M_{\odot}$ has a radius of the order of $13.7 \mathrm{~km}$, well within the values obtained by NICER [4,5] and other observations [56], and within or just slightly above the prediction obtained from terrestrial data [57], or the gravitational wave GW170817 [1,2] detected by LIGO-Virgo from a neutron-star merger [58-60]. We have calculated the tidal deformability of a canonical star with a mass $1.4 M_{\odot}$ according to Ref. [61]. The result obtained was $\Lambda_{1.4}=936-954$ depending on the hyperon content, well above the prediction of Ref. [2], $70<\Lambda_{1.4}<580$, which, however, was determined from a set of models that do not necessarily describe two-solar-mass stars. These high values of $\Lambda_{1.4}$ may indicate that the symmetry energy is too stiff, as discussed in Refs. [59,62], and the inclusion of a nonlinear $\omega-\rho$ term will soften the symmetry energy at high densities and decrease the value of $\Lambda_{1.4}$.

Within the present model we are able to describe NS as massive as the pulsar MSP J0740 + 6620 [9], in particular, if we constrain the optical potential of the $\Lambda$ hyperon in symmetric matter to experimental values. Only the hyperon fraction of baryonic matter and the value of the optical potential are sensitive to the precise value of $\delta$, for $\delta \geqslant 0.2$.

\section{CONCLUSIONS}

In the present study we have developed a QMC model based on the Bogoliubov quark model. The nucleons interact via the exchange of a scalar-isoscalar meson, a vectorisoscalar meson, and a vector-isovector meson. The baryon mass is derived from the baryon energy which includes $u$, $d$, and $s$ quarks. The parameters introduced at this level are chosen so that the vacuum constituent quark masses are

TABLE I. Properties of the stable neutron star with maximum mass, for several values of $\delta, M_{\max }, M_{\text {max }}^{b}, R, E_{0}, \rho^{c}, R_{1.4}, R_{1.6}, U_{\Lambda}\left(\rho_{0}\right)$, and $U_{\Xi}\left(\rho_{0}\right)$ are, respectively, the gravitational and baryonic masses, the star radius, the central energy density, the central baryonic density, the radius of neutron stars calculated for $1.4 M_{\odot}$ and $1.6 M_{\odot}$, and the optical potentials for a $\Lambda$ and $\Xi$ hyperon in symmetric nuclear matter at saturation.

\begin{tabular}{|c|c|c|c|c|c|c|c|c|c|c|}
\hline$\delta$ & $\begin{array}{l}M_{\max } \\
{\left[M_{\odot}\right]}\end{array}$ & $\begin{array}{l}M_{\max }^{b} \\
{\left[M_{\odot}\right]}\end{array}$ & $\begin{array}{c}\mathrm{R} \\
{[\mathrm{km}]}\end{array}$ & $\begin{array}{c}E_{0} \\
{\left[\mathrm{fm}^{-4}\right]}\end{array}$ & $u^{c}=\rho^{c} / \rho_{0}$ & $\begin{array}{c}R_{1.4} \\
{[\mathrm{~km}]}\end{array}$ & $\begin{array}{c}R_{1.6} \\
{[\mathrm{~km}]}\end{array}$ & $\begin{array}{l}U_{\Lambda}\left(\rho_{0}\right) \\
{[\mathrm{MeV}]}\end{array}$ & $\begin{array}{c}U_{\Sigma}\left(\rho_{0}\right) \\
{[\mathrm{MeV}]}\end{array}$ & $\begin{array}{c}U_{\Xi}\left(\rho_{0}\right) \\
{[\mathrm{MeV}]}\end{array}$ \\
\hline 0.0 & 1.97 & 2.28 & 10.91 & 7.25 & 7.674 & 13.731 & 13.492 & -75.34 & & -93.99 \\
\hline 0.05 & 2.08 & 2.43 & 11.42 & 6.43 & 6.882 & 13.752 & 13.680 & -67.57 & & -78.45 \\
\hline 0.1 & 2.16 & 2.53 & 11.73 & 5.97 & 6.429 & 13.750 & 13.693 & -59.80 & & -62.91 \\
\hline 0.15 & 2.20 & 2.58 & 11.83 & 5.84 & 6.285 & 13.746 & 13.698 & -52.03 & & -47.37 \\
\hline 0.25 & 2.21 & 2.60 & 11.84 & 5.82 & 6.255 & 13.746 & 13.696 & -36.49 & & -16.29 \\
\hline прен & 2.21 & 2.60 & 11.84 & 5.84 & 6.272 & 13.746 & 13.696 & & & \\
\hline прен QMC & 2.14 & 2.50 & 11.54 & 6.21 & 6.434 & 13.628 & 13.485 & & & \\
\hline YY QMC & 1.68 & 1.88 & 12.63 & 4.57 & 5.345 & 13.624 & 13.344 & -28.0 & +30.0 & -18.0 \\
\hline
\end{tabular}


reproduced. Hadronic matter is described by introducing a vector-isoscalar $\omega$ meson, which also includes an $\bar{s} s$ content, and a vector-isovector $b_{3}$ meson. To satisfy constraints imposed by neutron stars and hypernuclei it is shown that the coupling of the $\omega$ meson to the $s$ quark must be more repulsive than its coupling to the $u$ and $d$ quarks, and a parameter that takes this aspect into account has to be introduced, so that $\mathrm{SU}(3)$ symmetry is broken.

The couplings of the mesons to the nucleons were fixed so that nuclear matter properties, binding energy, saturation density, incompressibility, symmetry energy, and its slope at saturation, are adequately described. Once these parameters are fixed, only the parameter that defines how repulsive is the coupling of the $\omega$ meson to hyperons, remains to be fixed. Taking the optical potential of the $\Lambda$ hyperon of the order of $-30 \mathrm{MeV}$ as discussed in Refs. [40,63,64], no hyperons will be present inside a two-solar-mass star. A similar conclusion has been drawn in Ref. [55] where, within an auxiliary field diffusion Monte Carlo algorithm, it was shown that the three-body hyperon-nucleon interaction has an important role in softening the EoS at large densities. Using experimental separation energies of medium-light hypernuclei to constrain the $\Lambda N N$ force, they have shown that the onset of hyperons will occur above $0.56 \mathrm{fm}^{-3}$, and concluded that with the presently available experimental energies of $\Lambda$ hypernuclei it is not possible to draw a conclusive statement concerning the presence of hyperons inside neutron stars.

The present model predicts for the canonical neutron star a radius that is compatible with observations and predictions from the analysis of the GW170817 detection. The tidal deformability is, however, too large, and this may indicate that the symmetry energy is too stiff. A softer symmetry energy may be generated with the inclusion of a nonlinear $\omega-\rho$ term in the model [65].

\section{ACKNOWLEDGMENTS}

This work was partially supported by national funds from FCT (Fundação para a Ciência e a Tecnologia, I.P, Portugal) under the Projects No. UID/FIS/04564/2019, No. UID/04564/2020, and POCI-01-0145-FEDER-029912 with financial support from POCI, in its FEDER component, and by the FCT/MCTES budget through national funds (OE).
[1] B. P. Abbott et al. (Virgo, LIGO Scientific Collaborations), Phys. Rev. Lett. 119, 161101 (2017).

[2] B. P. Abbott et al. (LIGO Scientific and Virgo Collaborations), Phys. Rev. Lett. 121, 161101 (2018).

[3] B. P. Abbott et al. (LIGO Scientific, Virgo, Fermi GBM, INTEGRAL, IceCube, AstroSat Cadmium Zinc Telluride Imager Team, IPN, Insight-Hxmt, ANTARES, Swift, AGILE Team, 1M2H Team, Dark Energy Camera GW-EM, DES, DLT40, GRAWITA, Fermi-LAT, ATCA, ASKAP, Las Cumbres Observatory Group, OzGrav, DWF (Deeper Wider Faster Program), AST3, CAASTRO, VINROUGE, MASTER, J-GEM, GROWTH, JAGWAR, CaltechNRAO, TTU-NRAO, NuSTAR, Pan-STARRS, MAXI Team, TZAC Consortium, KU, Nordic Optical Telescope, ePESSTO, GROND, Texas Tech University, SALT Group, TOROS, BOOTES, MWA, CALET, IKI-GW Follow-up, H. E. S. S., LOFAR, LWA, HAWC, Pierre Auger, ALMA, Euro VLBI Team, Pi of Sky, Chandra Team at McGill University, DFN, ATLAS Telescopes, High Time Resolution Universe Survey, RIMAS, RATIR, SKA South Africa/MeerKAT), Astrophys. J. Lett. 848, L12 (2017).

[4] T. E. Riley, A. L. Watts et al., Astrophys. J. Lett. 887, L21 (2019).

[5] M. C. Miller et al., Astrophys. J. Lett. 887, L24 (2019).

[6] P. B. Demorest, T. Pennucci, S. M. Ransom, M. S. E. Roberts, and J. W. T. Hessels, Nature (London) 467, 1081 (2010).

[7] Z. Arzoumanian et al., Astrophys. J., Suppl. Ser. 235, 37 (2018).

[8] J. Antoniadis et al., Science 340, 1233232 (2013).

[9] H. T. Cromartie, E. Fonseca, S. M. Ransom et al., Nat. Astron. 4, 72 (2020).

[10] I. Bednarek, P. Haensel, J. L. Zdunik, M. Bejger, and R. Mańka, Astron. Astrophys. 543, A157 (2012).

[11] S. Weissenborn, D. Chatterjee, and J. Schaffner-Bielich, Phys. Rev. C 85, 065802 (2012); 90, 019904(E) (2014).

[12] S. Weissenborn, D. Chatterjee, and J. Schaffner-Bielich, Nucl. Phys. A 914, 421 (2013).
[13] G. Colucci and A. Sedrakian, Phys. Rev. C 87, 055806 (2013).

[14] C. Providência and A. Rabhi, Phys. Rev. C 87, 055801 (2013).

[15] E. N. E. van Dalen, G. Colucci, and A. Sedrakian, Phys. Lett. B 734, 383 (2014).

[16] M. Fortin, C. Providência, A. R. Raduta, F. Gulminelli, J. L. Zdunik, P. Haensel, and M. Bejger, Phys. Rev. C 94, 035804 (2016).

[17] J. D. Walecka, Ann. Phys. (NY) 83, 491 (1974).

[18] J. Boguta and A. R. Bodmer, Nucl. Phys. A 292, 413 (1977).

[19] H. Müller and B. D. Serot, Nucl. Phys. A 606, 508 (1996).

[20] S. Typel and H. H. Wolter, Nucl. Phys. A 656, 331 (1999).

[21] P. A. M. Guichon, Phys. Lett. B 200, 235 (1988).

[22] W. Bentz and A. W. Thomas, Nucl. Phys. A 696, 138 (2001).

[23] P. A. M. Guichon, K. Saito, E. Kodionov, and A. W. Thomas, Nucl. Phys. A 601, 349 (1996).

[24] K. Tsushima, K. Saito, J. Haidenbauer, and A. W. Thomas, Nucl. Phys. A 630, 691 (1998).

[25] P. A. M. Guichon and A. W. Thomas, Phys. Rev. Lett. 93, 132502 (2004).

[26] P. K. Panda, A. Mishra, J. M. Eisenberg, and W. Greiner, Phys. Rev. C 56, 3134 (1997).

[27] P. K. Panda, D. P. Menezes, and C. Providência, Phys. Rev. C 69, 025207 (2004).

[28] P. K. Panda, A. M. S. Santos, D. P. Menezes, and C. Providência, Phys. Rev. C 85, 055802 (2012).

[29] P. Yue and H. Shen, Phys. Rev. C 77, 045804 (2008).

[30] P. Yue, F. Yang, and H. Shen, Phys. Rev. C 79, 025803 (2009).

[31] J. Rikovska-Stone, P. A. M. Guichon, H. H. Matevosyan, and A. W. Thomas, Nucl. Phys. A 792, 341 (2007).

[32] P. A. M. Guichon, J. R. Stone, and A. W. Thomas, Prog. Part. Nucl. Phys. 100, 262 (2018).

[33] E. F. Batista, B. V. Carlson, and T. Frederico, Nucl. Phys. A 697, 469 (2002).

[34] N. Barik, R. N. Mishra, D. K. Mohanty, P. K. Panda, and T. Frederico, Phys. Rev. C 88, 015206 (2013). 
[35] R. N. Mishra, H. S. Sahoo, P. K. Panda, N. Barik, and T. Frederico, Phys. Rev. C 92, 045203 (2015).

[36] R. N. Mishra, H. S. Sahoo, P. K. Panda, N. Barik, and T. Frederico, Phys. Rev. C 94, 035805 (2016); 98, 019903(E) (2018).

[37] P. N. Bogolyubov, Ann. Inst. Henri Poincaré 8, 163 (1968).

[38] H. Bohr, S. A. Moszkowski, P. K. Panda, C. Providência, and J. da Providência, Int. J. Mod. Phys. E 25, 1650007 (2016).

[39] P. K. Panda, C. Providência, S. Moszkowski, and H. Bohr, Int. J. Mod. Phys. E 28, 1950034 (2019).

[40] A. Gal, E. V. Hungerford, and D. J. Millener, Rev. Mod. Phys. 88, 035004 (2016).

[41] S. L. Glashow, Phys. Rev. Lett. 11, 48 (1963).

[42] J. R. Stone and N. J. Stone, Phys. Rev. C 89, 044316 (2014).

[43] M. Oertel, M. Hempel, T. Klähn, and S. Typel, Rev. Mod. Phys. 89, 015007 (2017).

[44] N. Isgur and G. Karl, Phys. Rev. D 20, 1191 (1979).

[45] A. Le Yaouanc, L. Oliver, O. Pène, and J. C. Raynal, Phys. Rev. D 18, 1591 (1978).

[46] S. Capstick and W. Roberts, Prog. Part. Nucl. Phys. 45, S241 (2000).

[47] J. Haidenbauer and U.-G. Meißner, Nucl. Phys. A 936, 29 (2015).

[48] N. K. Glendenning and S. A. Moszkowski, Phys. Rev. Lett. 67, 2414 (1991).

[49] A. Bodmer, Phys. Rev. D 4, 1601 (1971).

[50] E. Witten, Phys. Rev. D 30, 272 (1984).

[51] R. C. Tolman, Phys. Rev. 55, 364 (1939).

[52] J. R. Oppenheimer and G. M. Volkoff, Phys. Rev. 55, 374 (1939).
[53] G. Baym, C. Pethick, and P. Sutherland, Astrophys. J. 170, 299 (1971).

[54] H. Pais and C. Providência, Phys. Rev. C 94, 015808 (2016).

[55] D. Lonardoni, A. Lovato, S. Gandolfi, and F. Pederiva, Phys. Rev. Lett. 114, 092301 (2015).

[56] P. Haensel, M. Bejger, M. Fortin, and J. L. Zdunik, Eur. Phys. J. A 52, 59 (2016).

[57] B.-A. Li and A. W. Steiner, Phys. Lett. B 642, 436 (2006).

[58] S. De, D. Finstad, J. M. Lattimer, D. A. Brown, E. Berger, and C. M. Biwer, Phys. Rev. Lett. 121, 091102 (2018).

[59] F. J. Fattoyev, J. Piekarewicz, and C. J. Horowitz, Phys. Rev. Lett. 120, 172702 (2018).

[60] T. Malik, N. Alam, M. Fortin, C. Providência, B. K. Agrawal, T. K. Jha, B. Kumar, and S. K. Patra, Phys. Rev. C 98, 035804 (2018).

[61] T. Hinderer, Astrophys. J. 677, 1216 (2008).

[62] V. Dexheimer, R. de Oliveira Gomes, S. Schramm, and H. Pais, J. Phys. G 46, 034002 (2019).

[63] H. Shen, F. Yang, annd H. Toki, Prog. Theor. Phys. 115, 325 (2006).

[64] D. Chatterjee and I. Vidaña, Eur. Phys. J. A 52, 29 (2016).

[65] C. J. Horowitz and J. Piekarewicz, Phys. Rev. Lett. 86, 5647 (2001).

Correction: A proof change request was misinterpreted by the production team and rendered errors in the affiliation indicators for the second and fourth authors. These affiliation indicators have been fixed. 